\title{
Tumor-infiltrating B cells and T cells correlate with postoperative prognosis in triple-negative carcinoma of the breast
}

\author{
Hajime Kuroda ${ }^{1,2^{*}}$, Tsengelmaa Jamiyan ${ }^{2,3}$, Rin Yamaguchi ${ }^{4}$, Akinari Kakumoto ${ }^{1,5}$, Akihito Abe ${ }^{6}$, Oi Harada ${ }^{7}$ and \\ Atsuko Masunaga ${ }^{1}$
}

\begin{abstract}
Background: In this study, we investigated CD20+ TILs in triple-negative breast cancer (TNBC) and their relationship with $\mathrm{T}$ lymphocyte subsets (CD4+, CD8+, CD25+, and FOXP3+), including their combined prognostic value using an immunohistochemical staining method.

Methods: We investigated 107 patients with TNBC for whom a full-face section stained by hematoxylin and eosin between 2006 and 2018 at Dokkyo Medical University Hospital was available.

Results: The strongest association of infiltrating CD20+ TILs was with CD4+ TILs. There was a significant relationship between CD20+ and CD4+ TILS $(r=0.177 ; p<0.001)$, CD8+ TILs $(r=0.085 ; p=0.002)$, and FOXP3+ TILs $(r=0.0043$; $p=0.032)$. No significant relationships were observed between the CD20+ and CD25+ TILs $(r=0.012 ; p=0.264)$. Multivariate analysis revealed that only the CD20+/FOXP3 ratio was an independent factor for relapse-free survival $(p<0.001)$ and overall survival $(p<0.001)$. Patients with tumors highly infiltrated by CD4+, CD8+, and CD20+ TILs had a good prognosis. In contrast, those with tumors weakly infiltrated by CD20+ TILs but highly infiltrated by CD25+ and FOXP3+ TILs had a poor prognosis.

Conclusions: CD20+ TILs may support an increase in CD4+ and CD8+ TILs, which altered the anti-tumor response, resulting in a positive prognosis. CD20+ TILs correlated with FOXP3+ Treg lymphocytes, which were reported to be correlated with a poor prognosis. Our study suggested that TIL-B cells have dual and conflicting roles in TIL-T immune reactions in TNBC.
\end{abstract}

Keywords: Breast, Triple-negative cancer, Tumor-infiltrating B lymphocytes, Tumor-infiltrating T lymphocytes

\section{Background}

Tumor-infiltrating lymphocytes (TILs) are a major factor in the tumor microenvironment, which is important in the development and progression of cancer. A correlation with prognosis in patients with breast cancer highly infiltrated by TILs was previously reported [1-3].

\footnotetext{
* Correspondence: kuroda.hajime@twmu.ac.jp

'Department of Diagnostic Pathology, Tokyo Women's Medical University, Medical Center East, 2-1-10 Nishiogu, Arakawa-ku, Tokyo 116-8567, Japan ${ }^{2}$ Department of Diagnostic Pathology, Dokkyo Medical University, Mibu, Japan

Full list of author information is available at the end of the article
}

Furthermore, several immunohistochemical studies concluded that tumor - infiltrating T lymphocytes (TILs-T) have antitumor activity in mammary glands. Most TILs in cancer are $\mathrm{T}$ lymphocytes, and include $\mathrm{CD} 4+$ (helper) and CD8+ (cytotoxic) lymphocytes [4-9]. CD4+ TILs are necessary to activate proliferation and memory in tumor-specific CD8+ TILs [10]. We previously found that CD4+ and CD8+ TILs are correlated with a favorable prognosis in triple-negative breast cancer (TNBC) [11]. Although the prognostic correlation of TILs-T has been widely reported, there are few studies on

(C) The Author(s). 2021 Open Access This article is licensed under a Creative Commons Attribution 4.0 International License, which permits use, sharing, adaptation, distribution and reproduction in any medium or format, as long as you give appropriate credit to the original author(s) and the source, provide a link to the Creative Commons licence, and indicate if changes were made. The images or other third party material in this article are included in the article's Creative Commons licence, unless indicated otherwise in a credit line to the material. If material is not included in the article's Creative Commons licence and your intended use is not permitted by statutory regulation or exceeds the permitted use, you will need to obtain permission directly from the copyright holder. To view a copy of this licence, visit http://creativecommons.org/licenses/by/4.0/ The Creative Commons Public Domain Dedication waiver (http://creativecommons.org/publicdomain/zero/1.0/) applies to the data made available in this article, unless otherwise stated in a credit line to the data. 
infiltrating B lymphocytes (TILs-B) in breast cancer and there is no consensus on their prognostic impact [1220]. In addition, few studies have investigated TILs-B and TILs-T in combination [21-24]. Indeed, TILs that contain both TIL-B and TIL-T are correlated with lymphocyte proliferation and a good prognosis, suggesting that TIL-B cooperate with TIL-T in an anti-tumor reaction [25]. Among $\mathrm{T}$ lymphocytes, $\mathrm{T}$ regulatory (Treg) lymphocytes suppress effector $\mathrm{T}$ lymphocytes and control immune responses [26]. The mechanisms governing Treg lymphocyte proliferation and function recently attracted attention because of their importance in suppressing the expansion of autoimmunity and their therapeutic viability. In animal models, immune-related tumor clearance and heightened response to immunotherapy were improved by the removal of Treg lymphocytes [27]. Furthermore, high infiltration of Treg lymphocytes was reported to be correlated with a negative prognosis in human cancers, including breast cancer, and they may represent a new therapeutic target [26-31]. Forkhead box protein 3 (FOXP3) and CD25, which are considered the most reliable markers of Treg lymphocytes play an important role in immunesuppression, thereby inducing immune tolerance. However, TIL-B and Treg lymphocyte involvement in human cancer remains unclear.

The correlation between CD20+ TILs and TNBC has not been clarified, and their relationship with other immune cell subsets (CD4+, CD8+, CD25+, FOXP3+) in TNBC has not been reported. This study was carried out to evaluate the behavior of CD20+ TILs in TNBC and their relationship with $\mathrm{T}$ lymphocyte subsets (CD4+, CD8+, CD25+, FOXP3+), assessing their prognostic value using an immunohistochemical staining method.

\section{Materials and methods Patients}

We investigated 107 patients with TNBC for whom a full-face section stained by hematoxylin and eosin (H\&E) between 2006 and 2018 at Dokkyo Medical University Hospital was available. All patients received preoperative chemotherapy. The clinicopathological variables, including age at the time of diagnosis, tumor size, histology, tumor grade, Mib-1 index, and TILs status, were reviewed.

\section{Immunohistochemistry (IHC)}

IHC was performed using monoclonal antibodies against estrogen receptor (ER) (clone SP1, Novocastra (Leica), prediluted, nuclear), progesterone receptor (PgR) (clone 1E2, Novocastra (Leica), prediluted, nuclear), human epidermal growth factor receptor 2 (HER2) (clone 4B5, Roche (Ventana), prediluted, membranous), CD4 (CD4, clone 1F6, Novocastra (Leica), 1:40), CD8 (CD8, clone
4B11, Novocastra (Leica), prediluted), CD20 (CD20, clone L26, Nichirei), CD25 (CD25, clone 4C9 Novocastra (Leica), prediluted), and FOXP3 (FOXP3, clone 236A/E7, Abcam, 1:50). Hematoxylin was used as a counterstain. ER and PgR expression was estimated, and $>1 \%$ nuclear-stained tumor cells was considered positive according to the American Society of Clinical Oncology and the College of American Pathologist (ASCO/CAP) guidelines [32]. HER2-negative status was confirmed as a staining score of $0 / 1+$. In cases with an IHC score of 2, fluorescence in situ hybridization (FISH) was used to determine the HER2 status, and it was considered positive when the ratio of HER2 to chromosome enumeration probe 17 (CEP17) was $>2.0$ [33]. The mib1 index was estimated using a semiquantitative visual method and the threshold was set at $20 \%$ based on a previous report [34]. Tumor cells were considered positive for mib-1 only when nuclear staining was notable. TILs in H\&E-stained sections were assessed according to the International Immuno-Oncology Biomarkers Working Group [35] and the threshold was set at 30\% [36]. Stromal TILs were defined as the percentage of immune cells in the tumor stroma and outside the tumor nests. To evaluate CD4+, CD8+, CD20+, CD25+, and FOXP3+ TILs, the number of positive cells was calculated from IHC staining. Each specimen was screened at low magnification $(\times 100)$, and the five areas with the greatest number of positively stained cells in the stroma were selected for further analysis. The mean TIL count in these areas in each case was estimated at high $(\times 400)$ magnification. Moreover, the ratios of CD20/CD4, CD20/CD8, CD20/CD25, and CD20/FOXP3 were calculated. Statistically, the number of positive cells was classified into low and high groups based on a threshold assessed by the median (Table 1). Each specimen was analyzed by two investigators (TJ and $\mathrm{HK}$ ) who were blinded to the clinicopathological information, and an average of the results was obtained.

\section{Statistical analysis}

The correlation between the number of immune cells (CD4+, CD8+, CD20+, CD25+, and FOXP3+) and the clinicopathological variables was analyzed using the chi

Table 1 Distribution pattern of stromal TILS in TNBC

\begin{tabular}{lllll}
\hline Variables & Mean & SE & Median & Range \\
\hline CD4+ TILs & 105.07 & 5.56 & 104 & 273 \\
CD8+ TILS & 91.79 & 5.02 & 81 & 251 \\
CD20+ TILs & 79.43 & 7.19 & 60 & 276 \\
CD25+ TILs & 21.47 & 1.49 & 16 & 66 \\
FOXP3+ TILs & 38.27 & 2.89 & 31 & 153 \\
\hline
\end{tabular}

TILs tumor-infiltrating lymphocytes, TNBC triple-negative breast cancer, SE standard error 
square test. The Wilcoxon signed ranks test was performed to compare the $\mathrm{B}$ and $\mathrm{T}$ cell markers. We explored the association between CD20 and T cell markers using a linear regression model. The Kaplan-Meier method was used to estimate relapse-free survival (RFS) and overall survival (OS), and differences were analyzed by the log-rank test. Univariate and multivariate Cox proportional hazard models were used to assess the hazard ratios (HRs) with a confidence interval (CI) of 95\% for survival and $p<0.05$ was considered significant. We converted continuous variables into categorical variables in the Cox regression model, which was adjusted for relevant clinical covariates, including age, tumor size, histological grade, and lymph node status. Statistical analyses were carried out using IBM SPSS Statistics 26 (IBM, Armonk, NY, United States).

\section{Results}

We investigated the populations of infiltrating immune cells in TNBC. Immune cell subsets were distinguished by staining of CD4 (T helper lymphocytes), CD8 (cytotoxic T lymphocytes), CD20 (B lymphocytes), and CD25 or FOXP3 (Treg lymphocytes) (Fig. 1). The strongest association of infiltrating CD20+ TILs was with CD4+ TILs (Fig. 2). There was a significant relationship between CD20+ and CD4+ TILs $\left(\mathrm{r}^{2}=0.177 ; p<0.001\right)$, CD8+ TILs $\left(\mathrm{r}^{2}=0.085 ; p=0.002\right)$, and FOXP3+ TILs $\left(\mathrm{r}^{2}=0.043 ; p=0.032\right)$. However, no significant relationships were observed between the CD20+ and CD25+ TILs $\left(r^{2}=0.012 ; p=0.264\right)$ (Fig. 2). The correlation with CD20+ TILs of the total number of CD4+, CD8+, CD25+, and FOXP3+ TILs $(p<0.001, p=0.04, p<$ 0.001 , and $p<0.001)$ in patients with TNBC was
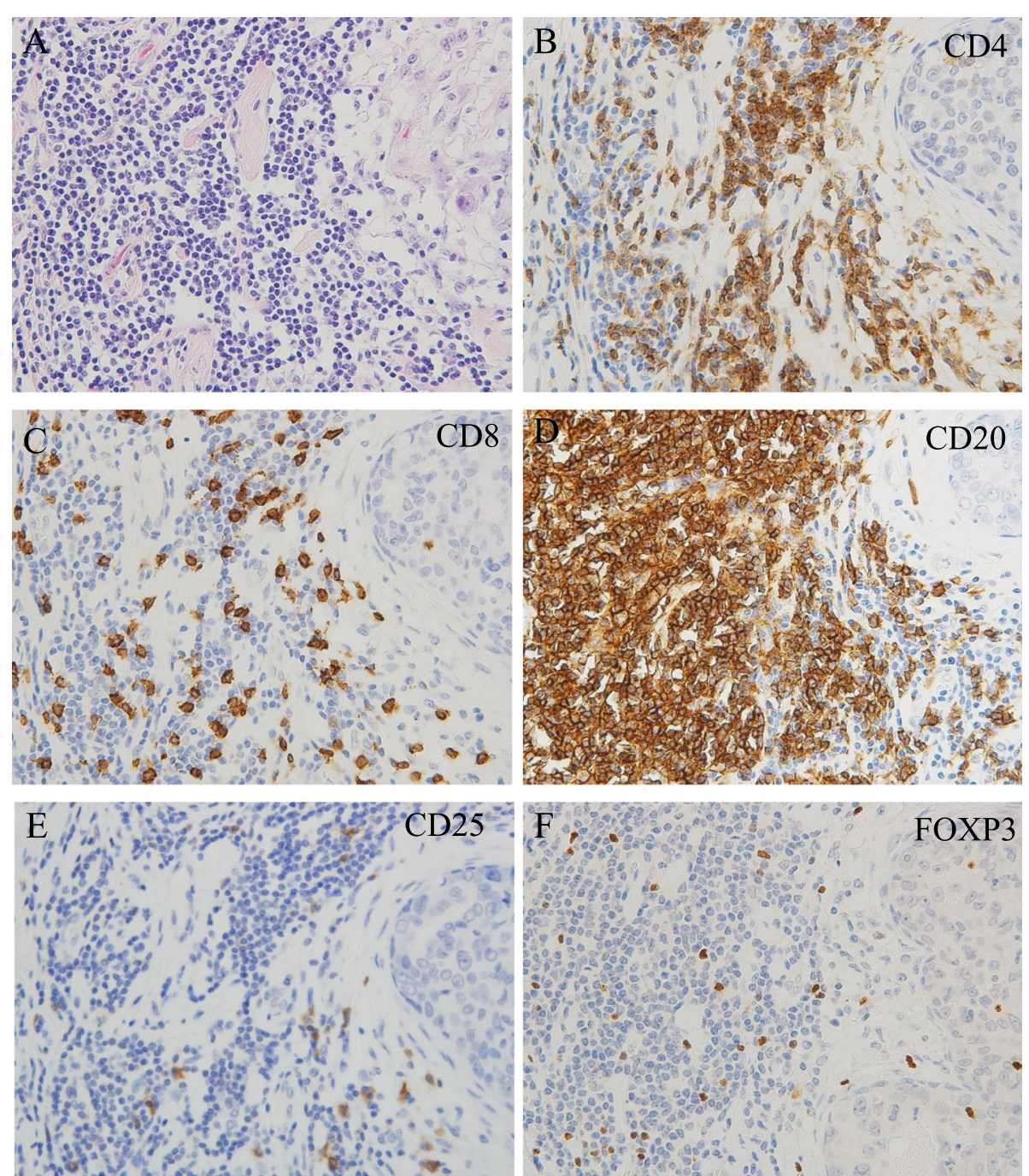

Fig. 1 Representative photomicrographs of TILs in patients with TNBC. A patient with a high level of TILs on HE staining (a). IHC using primary antibodies against CD4 (b), CD8 (c), CD20 (d), CD25 (e), and FOXP3 (f) to characterize TILs in the same section of tumor tissue (original magnification, $\times 400$ ) 
Scatter plot and regression line of CD20 and CD4. $p<0.001$

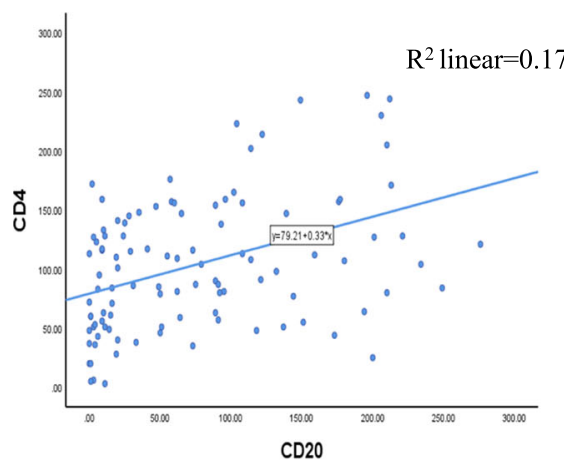

Scatter plot and regression line of CD20 and CD25 $p<0.264$

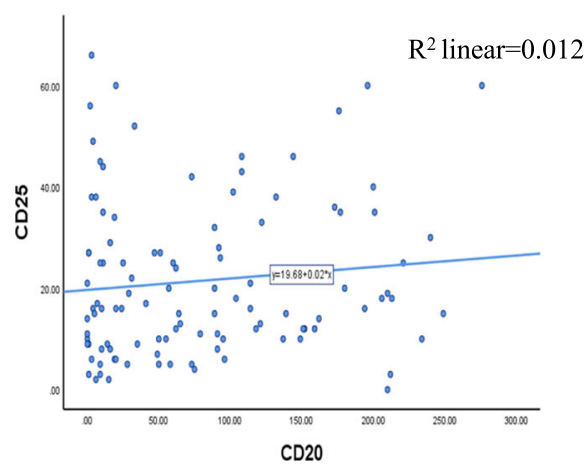

Scatter plot and regression line of

CD20 and CD8. $p=0.002$

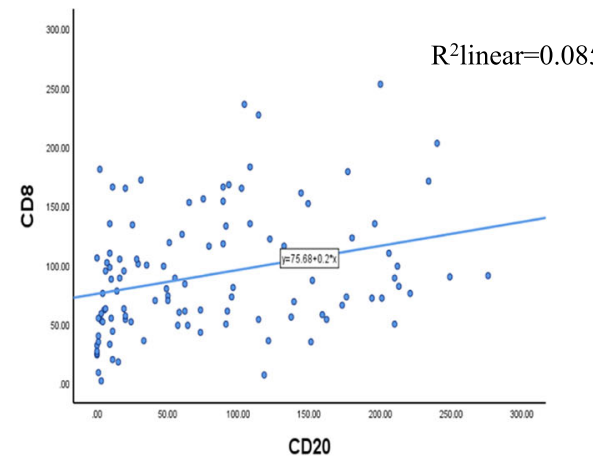

Scatter plot and regression line of CD20 and FOXP3 $p<0.032$

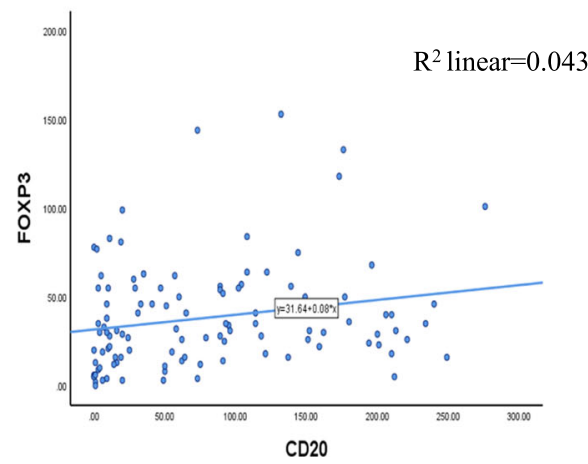

Fig. 2 Absolute counts of CD20+ TLLs and CD4+, CD8+, CD25, and FOXP3+ TLL in the peripheral stroma of patients with TNBC. The horizontal and vertical reference lines display absolute numbers of CD20+ TlLs and CD4+, CD8+, CD25+, and FOXP3+ TlLs. As shown by the regression lines, counts of CD4+, CD8+, and FOXP3+ TLLs increased along with CD20+ TLLs in TNBC. However, the counts of CD25+ were not related to CD20+ TLLS

examined (Fig. 3). Overall, T helper lymphocytes were the main immune cells infiltrating the TNBC stromal region, and these included cytotoxic T lymphocytes, B lymphocytes, and Treg lymphocytes. Infiltration of CD20+ TILs was separated into low and high groups to adapt to the median threshold, and analyzed for correlations with the clinicopathological features (Tables 1 and 2). As a result, the threshold was 104, 81, 60, 16, and 31 for CD4, CD8, CD20, CD25, and FOXP3, respectively. A high density of CD20+ TILs was significantly related to a high density of TILs $(p<0.001)$. However, there was no significant relationship between a high density of CD20+ TILs and other clinicopathological characteristics (age at the time of diagnosis, tumor size, tumor grade, histology, lymph node metastasis, and mib-1 index).

Next, we analyzed the correlation of infiltrating CD20+ TILs with RFS $(p=0.007)$ and OS $(p=0.004)$ in patients with TNBC. Patients with tumors highly infiltrated by CD20+ TILs had a good prognosis (Fig. 4). We analyzed the prognostic value of CD20 + TILs compared with other investigated immune cell subsets (Table 3). In univariate survival analyses, the $\mathrm{CD} 20+/ \mathrm{CD} 4+$ and CD20+/CD8+ ratios were not significantly associated with RFS ( $p=0.348$, and $p=0.319)$ or OS $(p=0.406$ and $p=0.274)$. However, lower CD20+/CD25+ and/or CD20+/FOXP3 ratios were correlated with a poorer RFS $(p=0.009$, and $p=0.001)$ and OS $(p=0.007$ and $p=$ 0.001 ). By multivariate analysis, only a lower $\mathrm{CD} 20+$ / FOXP3+ ratio was an independent factor for a poorer RFS $(p<0.001)$ and OS $(p<0.001)$.

To analyze the prognostic value of TILs-B in correlation with infiltrating TILs-T, we investigated the prognostic impact of groups of CD20+ TILs (high or low) compared with that of CD4+, CD8+, CD25+, and FOXP3+ TILs (high or low). Patients with tumors highly infiltrated by CD4+ and CD20+ TILs had a better prognosis than those with tumors highly infiltrated by CD4+ TILs but weakly infiltrated by CD20+ TILs (Fig. 5a and b). In patients with tumors weakly infiltrated by CD4+ TILs, the prognostic value of CD20+ TILs decreased. 


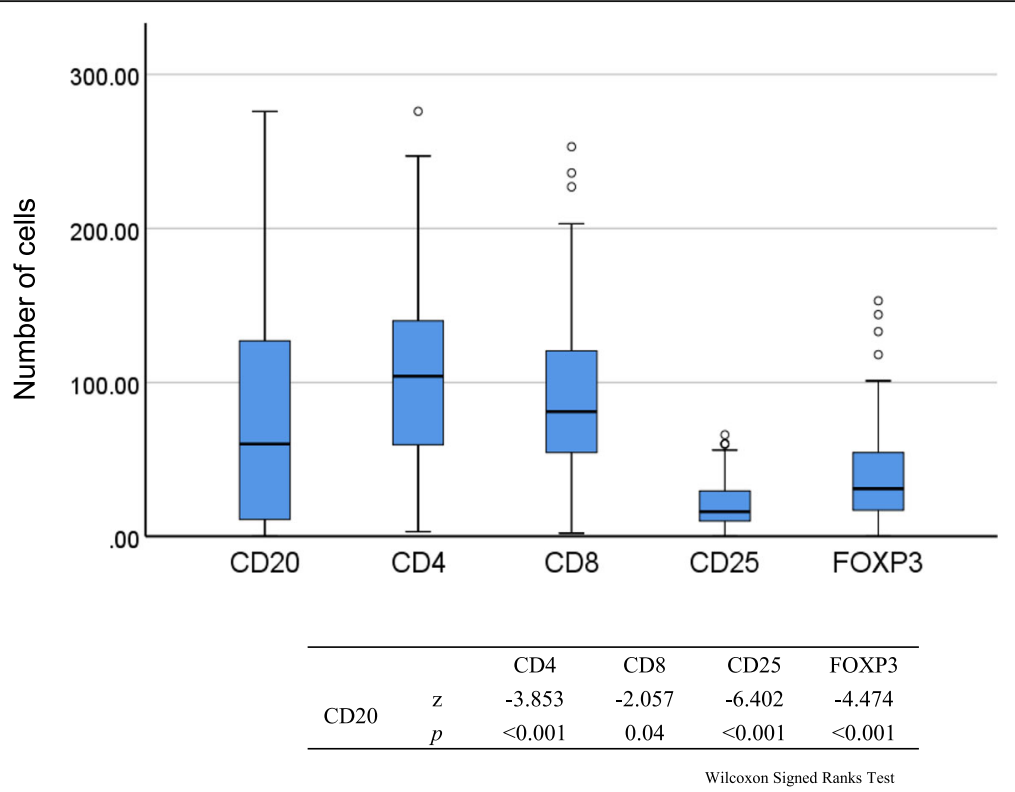

Fig. 3 Box plots displaying the correlation of CD20+ TILs with the absolute numbers of CD4+, CD8+, FOXP3+, and CD25 TILs in patients with TNBC. The bars are median values, the box displays the interquartile range (25-75\%), and the whiskers extend to $1.5 \times$ the interquartile range

Table 2 Clinicopathological characteristics of TNBC and the status of stromal CD20 $(n=107)$

\begin{tabular}{|c|c|c|c|c|}
\hline \multirow{2}{*}{$\begin{array}{l}\text { Clinicopathological } \\
\text { characteristics }\end{array}$} & \multirow{2}{*}{$\begin{array}{l}\text { Total no. } \\
\text { of cases }\end{array}$} & \multicolumn{3}{|c|}{ CD20 } \\
\hline & & Low & High & $P$ value \\
\hline Age (years) & & & & 0.499 \\
\hline$<60$ & 53 & 25 & 28 & \\
\hline$\geq 60$ & 54 & 29 & 25 & \\
\hline Tumor size $(\mathrm{cm})$ & & & & 0.087 \\
\hline$\leq 2$ & 66 & 29 & 37 & \\
\hline$>2$ & 41 & 25 & 16 & \\
\hline Tumor grade & & & & 0.564 \\
\hline$|\&| \mid$ & 31 & 17 & 14 & \\
\hline III & 76 & 37 & 39 & \\
\hline Histology & & & & 0.806 \\
\hline IDC & 82 & 40 & 42 & \\
\hline ILC & 2 & 1 & 1 & \\
\hline other types & 23 & 13 & 10 & \\
\hline Lymph node metastasis & & & & 0.952 \\
\hline absent & 77 & 39 & 38 & \\
\hline present & 30 & 15 & 15 & \\
\hline Mib-1 index & & & & 0.475 \\
\hline$<20 \%$ & 19 & 11 & 8 & \\
\hline$\geq 20 \%$ & 88 & 43 & 45 & \\
\hline TILS & & & & $<0.001$ \\
\hline low & 58 & 44 & 14 & \\
\hline high & 49 & 10 & 39 & \\
\hline
\end{tabular}

TNBC triple-negative breast cancer, IDC invasive ductal carcinoma, ILC invasive lobular carcinoma, TILs Tumor-infiltrating lymphocytes X2 test
Almost identical correlations were noted between CD20+ TIL infiltration and CD8+ TIL infiltration (Fig. $5 \mathrm{c}$ and $\mathrm{d})$. Of note, in patients with tumors weakly infiltrated by CD20+ TILs but highly infiltrated by CD25+ and FOXP3+ TILs, the opposite effect was observed (Fig. 5e-h).

\section{Discussion}

In this study, we investigated TILs-B in TNBC and their relationships with $\mathrm{T}$ lymphocyte subsets. We examined the prognostic role of groups of CD20 (high and low) combined with those of CD4 (high or low) and CD8 (high or low) in the stromal component using KaplanMeier curves. CD20+ TILs were associated with CD4+ and CD8+ TILs. A good prognosis was observed in TNBC in which tumors were highly infiltrated by CD20+ TILs combined with CD4+ and CD8+ TILs; therefore, CD20+ TILs may support CD4+ and CD8+ TILs in altering the anti-tumor response.

Correlations of TILs-B with TILs-T were previously reported in liver, ovary, and colon carcinomas [21-24]. Shi et al. used IHC to examine the population and prognostic significance of CD20+ TILs in a hepatocellular carcinoma series [23]. A high density of TILs-B in the tumor margin induced an increase in stromal CD8+ TILs. Nielsen et al. also reported an association between stromal CD20+ and CD8+ TILs in ovarian carcinoma [21]. Recently, CD20+ TILs were reported to be highly and positively correlated with CD8+ TILs in large colon cancer series [24]. As mentioned above, there are several reports of an association between TIL-B and CD8+ TILs 


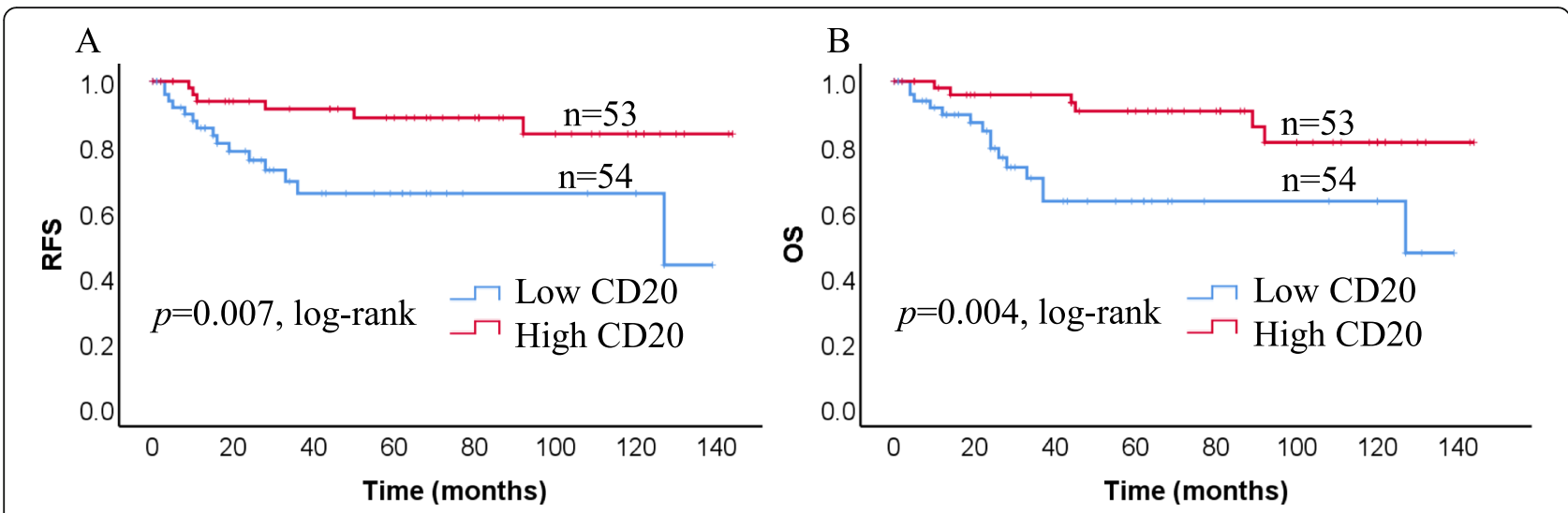

Fig. 4 Kaplan-Meier plots showing RFS and OS in TNBC according to infiltration of CD20+ TILs. Log-rank tests were used to estimate $P$-values

in human diseases, including cancer. However, there are few reports on TILs-B and their influence on CD4+ TILs. Similar to CD8+ TILs, CD20+ TILs were reported to be associated with CD4+ TILs in non-small cell lung cancer [37]. B lymphocytes can support anti-tumor activity through several mechanisms. This concept is supported by studies demonstrating that deficient $\mathrm{T}$ cell responses are correlated with $\mathrm{B}$ cell deficiency in genedisrupted mice [38, 39]. B lymphocytes from tumorbearing or naïve donors played similar roles in suppressing tumor growth when transplanted with $\mathrm{T}$ lymphocytes [39]. Furthermore, in B lymphocyte-deficient mice produced by continuous injection of anti B antibodies, $\mathrm{T}$ lymphocytes activated in lymph nodes relied on antigen presentation by B lymphocytes. This suggests that B lymphocytes capture antigens and present them to $\mathrm{T}$ lymphocytes after transplantation into the recipient mice. B lymphocytes are assumed to bind tumor antigens through surface immunoglobulin (Ig) molecules, process them, and then present tumor-derived antigens through major histocompatibility complex classes I and II to induce the activation of both CD8+ cells and CD4+ T lymphocytes [40].

In vivo, coordinated $\mathrm{B}$ and $\mathrm{T}$ lymphocyte reactions were previously reported in both auto-immunity and allograft rejection [41, 42]. In allograft rejection and chronic infection, B lymphocytes are observed in the central tertiary lymphoid structures in affected tissues [43]. In a xenograft model of rheumatoid arthritis, the loss of B lymphocytes reduced $\mathrm{T}$ lymphocyte activation and proliferation in stroma according to anti-CD20 antibody staining. Furthermore, the loss of $\mathrm{T}$ lymphocytes induced the breakdown of tertiary lymphoid structures and depletion of Ig immunoglobulin production by B lymphocytes [44]. These reports support our results that TILs-B are associated with stroma CD4+ and CD8+ TILs in TNBC. TILs-B may induce Treg lymphocyte development and proliferation, and affect the prognosis. In our study, CD20+ TILs were associated with FOXP3+ TILs expression, but not with CD25+ TILs. However, in the multivariate analysis, CD20+ and FOXP3+ TILs had an opposing relationship. Furthermore, CD25+ and FOXP3+ TILs had no prognostic advantage based on Kaplan-Meier curves. High infiltration of Treg lymphocytes was reported to correlate with the prognosis in human cancers [11, 26-31]. We previously reported that TNBC is associated with FOXP3+ TILs [11]. In addition, the distribution of circulating $\mathrm{CD} 4+\mathrm{CD} 25+\mathrm{FOXP} 3+$ Treg lymphocytes and CD19+CD24 + CD 38 + B lymphocytes significantly increased in breast cancer patients compared with healthy controls using blood samples [45]. CD19+ B lymphocytes induced the expansion of Treg lymphocytes expressing FOXP3 in in vitro assays [46]. Furthermore, stimulated B lymphocytes were used

Table 3 Cox regression analyses of infiltrating immune cells in RFS and OS of TNBC patients

\begin{tabular}{|c|c|c|c|c|c|c|c|c|c|c|c|c|}
\hline \multirow{3}{*}{$\begin{array}{l}\text { Immune } \\
\text { cell } \\
\text { markers }\end{array}$} & \multicolumn{6}{|c|}{ Univariate analysis } & \multicolumn{6}{|c|}{ Multivariate analysis } \\
\hline & \multicolumn{3}{|c|}{ RFS } & \multicolumn{3}{|l|}{ OS } & \multicolumn{3}{|c|}{ RFS } & \multicolumn{3}{|l|}{ OS } \\
\hline & HR & 95\% Cl & $P$ value & $\mathrm{HR}$ & $95 \% \mathrm{Cl}$ & $P$ value & HR & 95\% Cl & $P$ value & HR & $95 \% \mathrm{Cl}$ & $P$ value \\
\hline CD20/CD4 & 1.618 & $0.592-4.419$ & 0.348 & 1.532 & $0.560-4.190$ & 0.406 & 2.797 & $0.855-9.151$ & 0.089 & 1.898 & $0.557-6.467$ & 0.306 \\
\hline CD20/CD8 & 0.643 & $0.270-1.532$ & 0.319 & 0.615 & $0.258-1.468$ & 0.274 & 2.915 & $0.941-9.031$ & 0.064 & 3.147 & $0.936-10.57$ & 0.064 \\
\hline CD20/CD25 & 0.261 & $0.095-0.717$ & 0.009 & 0.247 & $0.090-0.678$ & 0.007 & 1.082 & $0.303-3.857$ & 0.903 & 0.694 & $0.191-2.527$ & 0.58 \\
\hline CD20/FOXP3 & 0.085 & $0.020-0.367$ & 0.001 & 0.083 & $0.019-0.361$ & 0.001 & 0.028 & $0.005-0.166$ & $<0.001$ & 0.044 & $0.008-0.246$ & $<0.001$ \\
\hline
\end{tabular}

RFS relapse-free survival, OS overall survival, TNBC triple-negative breast cancer, $\mathrm{HR}$ hazard ratio, $\mathrm{Cl}$ confidence interval 

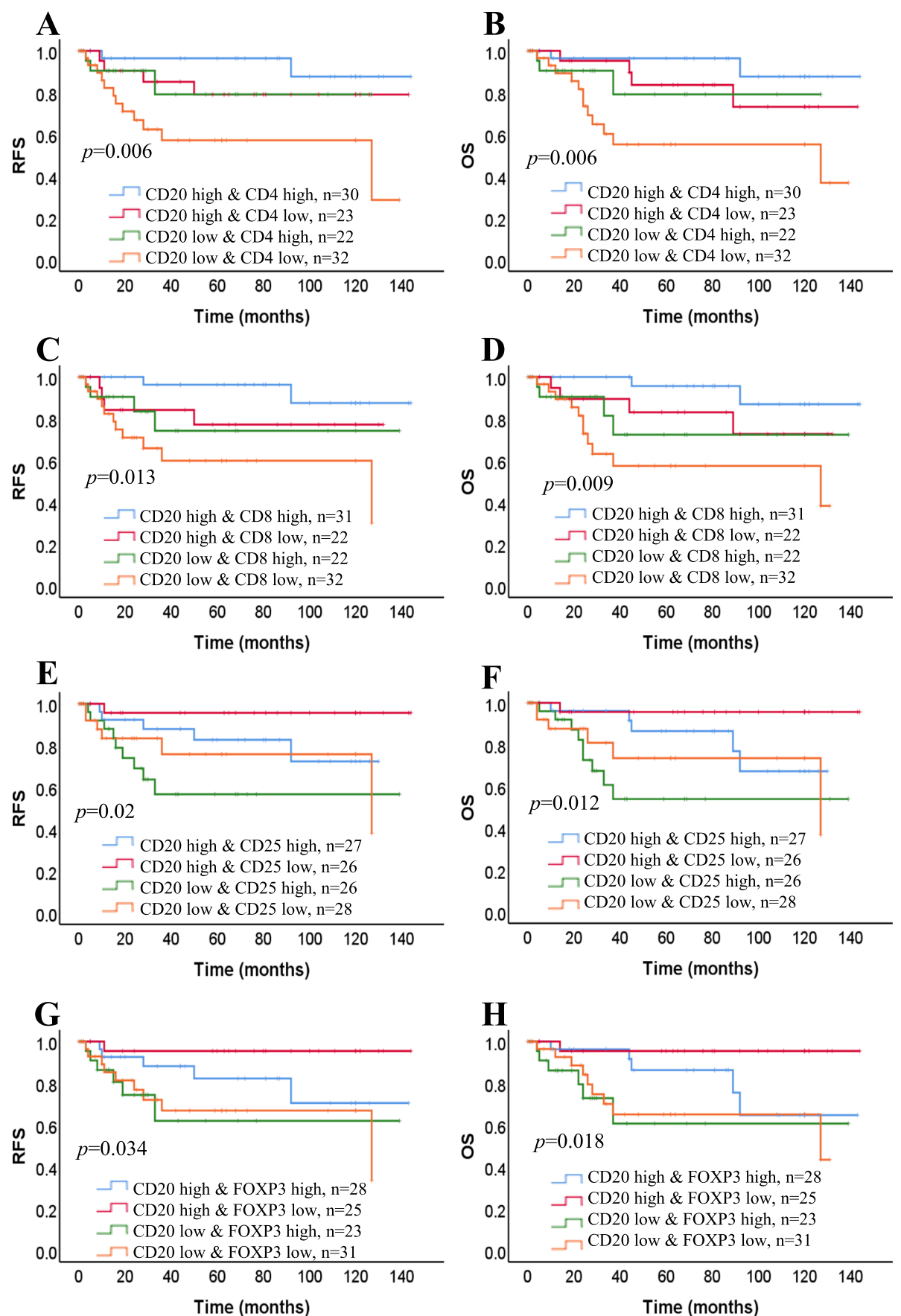

Fig. 5 Densities of TIL-B and TIL-T are associated with patient survival. Kaplan-Meier curves showing RFS and OS of the patients by combining CD20+ with CD4+, CD8+, CD25+, and FOXP3+ TILs. a, b CD20-high CD4-high ( $n=30)$, CD20-high CD4-low ( $n=23)$, CD20-low CD4-high ( $n=22)$, CD20-low CD4-low $(n=32)$. c, d CD8-high CD4-high $(n=31)$, CD20-high CD8-low $(n=22)$, CD20-low CD8-high $(n=22)$, CD20-low CD8-low $(n=$ 32). e, f CD20-high CD25-high $(n=27)$, CD20-high CD25-low $(n=26)$, CD20-low CD25-high $(n=26)$, CD20-low CD25-low $(n=28)$. $\mathbf{g}, \mathbf{h} C D 20$-high FOXP3-high $(n=28)$, CD20-high FOXP3-low $(n=25)$, CD20-low FOXP3-high $(n=23)$, CD20-low FOXP3-low $(n=31)$. Graph shows $p=$ log-rank test $P$-values

to induce Treg lymphocytes [47]. Co-culture of CD40Lactivated B lymphocytes resulted in an increased number of CD4+ T lymphocytes that also expressed CD25 and FOXP3. These Treg lymphocytes inhibited the development of $\mathrm{T}$ lymphocytes by reacting with the original target alloantigens expressed on the B lymphocytes. This tolerance theory is reasonable based on our study demonstrating that CD20+ TILs are correlated with a 
positive prognosis and increase the number of FOXP3+ Treg lymphocytes. Therefore, our study suggested that TILs- $\mathrm{B}$ have dual and conflicting roles in TILs- $\mathrm{T}$ immune reactions in TNBC.

However, the counts of CD25+ TILs, which are also considered to be a Treg lymphocyte marker, were not related to CD20+ TILs. FOXP3 is considered to be the most reliable Treg lymphocyte marker in human cancer $[48,49]$. However, it is expressed by Treg lymphocytes, epithelial tumor cells, and primed CD4+ and CD8+ lymphocytes. Therefore, several previous studies included CD25+ as a marker of Treg lymphocytes. Although CD25 is expressed by effector $\mathrm{T}$ lymphocytes like FOXP3, it is unclear whether CD25 more accurately estimates Treg lymphocytes. Based on previous reports, the differences in Treg lymphocytes may be due to the marker used.

There are some limitations to this study. We found only a weak-fair correlation by scatter plot and the regression line among CD4, CD8, CD25, FOXP3, and CD20 expression. A possible explanation is the small number of TNBC cases examined in this study. The low $r^{2}$ graphs show that even noisy, high - variability data can have a significant tendency. However, the tendency indicates that CD20 still influences CD4, CD8, and FOXP3. Therefore, the outcome would likely be clearer if a greater number of TNBCs were examined.

Another limitation is that we used a visual assessment of TILs. However, there is no standard visual assessment estimation method for TILs as compared with a digital assessment system. At present, the visual assessment continues to have inherent limitations that cannot be fully addressed through standardization and training. In recent years, some researchers have estimated TILs by digital assessment systems. However, they also have some limitations. The International ImmunoOncology Biomarker Working Group recommended that digital pathology algorithms need to account for the complexity involved in TIL-scoring procedures by a pathologist [50]. However, even validated standalone digital assessment requires checks by pathologists to prevent unexpected failures. Further, a Wouters et al. meta-analysis article reported scoring of CD20+ TIL either visually or digitally using various software packages and found no differences in the direction of the outcome data for either of these methods [51]. Therefore, it is unavoidable that there is some ambiguity with all methods. Currently, pathologists need to accept that there is some ambiguity in terms of estimation.

\section{Conclusion}

Our study provides evidence of a correlation between TILs-B and TILs-T in TNBC patients. CD20+ TILs were associated with CD4+, CD8+ and FOXP3+ TILs, but not with CD25+ TILs. A good prognosis was observed for patients with tumors that were highly infiltrated by CD20+ combined with CD4+ and CD8+, suggesting that CD20+ TILs support a CD4+ and CD8+ TIL-altered anti-tumor response. However, CD25+ and FOXP3+ Treg lymphocytes had no prognostic advantage based on Kaplan-Meier curves. Moreover, in multivariate analysis, CD20+ and FOXP3+ TILs had an opposite effect on RFS and OS. CD20+ TILs were correlated with a positive prognosis, and increased CD4+ and CD8+ TILs and FOXP3+ Treg lymphocytes. Therefore, our study suggested that TILs-B have dual and conflicting roles in TILs-T immune reactions in TNBC.

\section{Abbreviations}

TILs: Tumor-infiltrating lymphocytes; TILs-T: Tumor-infiltrating T lymphocytes; TNBC: Triple-negative breast cancer; TILs-B: Tumor-infiltrating B lymphocytes; Treg: T regulatory; FOXP3: Forkhead box protein 3; H\&E: Hematoxylin and eosin; IHC: Immunohistochemistry; ER: Estrogen receptor; PgR: Progesterone receptor; HER2: Human epidermal growth factor receptor 2; ASCO/CAP: The American Society of Clinical Oncology/College of American Pathologists; FISH: Fluorescence in situ hybridization; CEP17: Chromosome enumeration probe 17; RFS: Relapse-free survival; OS: Overall survival; HRs: Hazard ratios; Cl: Confidence interval

\section{Acknowledgments}

The authors thank Chiaki Matsuyama and Ayako Shimizu for their advice and technical assistance with $\mathrm{HC}$ staining.

\section{Authors' contributions}

H Kuroda and T Jamiyan contributed equally to this work; A Abe collected clinical information; $\mathrm{H}$ Kuroda and T Jamiyan reviewed the pathological diagnosis; $\mathrm{H}$ Kuroda and $\mathrm{T}$ Jamiyan analyzed the data and wrote the manuscript; R Yamaguchi, A Kakumoto, O Harada, and A Masunaga critically revised the manuscript; $\mathrm{H}$ Kuroda and $\mathrm{T}$ Jamiyan designed the study; $\mathrm{H}$ Kuroda gave the final approval of the manuscript for publication. All authors read and approved the final manuscript.

\section{Funding}

We have no funding sources to report.

\section{Availability of data and materials}

The datasets used and/or analyzed during the current study available from the corresponding author on reasonable request.

\section{Declarations}

Ethics approval and consent to participate

This study was conducted according to the Declaration of Helsinki and after approval by the ethics committee of Dokkyo Medical University (No.28009).

All patients provided written informed consent prior to study participation.

Consent for publication

All authors agreed to the publication.

\section{Competing interests}

The authors declare that they have no competing interests.

\section{Author details}

'Department of Diagnostic Pathology, Tokyo Women's Medical University, Medical Center East, 2-1-10 Nishiogu, Arakawa-ku, Tokyo 116-8567, Japan. ${ }^{2}$ Department of Diagnostic Pathology, Dokkyo Medical University, Mibu, Japan. ${ }^{3}$ Department of Pathology and Forensic Medicine, Mongolian National University of Medical Sciences, Ulan Bator, Mongolia. ${ }^{4}$ Department of Pathology \& Laboratory Medicine, Kurume University Medical Center, Kurume, Japan. ${ }^{5}$ Department of Diagnostic Pathology, Nasu Red Cross 
Hospital, Otawara, Japan. ${ }^{6}$ Breast Center, Dokkyo Medical University, Mibu, Japan. ${ }^{7}$ Breast center, Showa University, Tokyo, Japan.

\section{Received: 14 November 2020 Accepted: 3 March 2021 Published online: 16 March 2021}

\section{References}

1. Mersin H, Yildirim E, Berberoglu U, Gulben K. The prognostic importance of triple negative breast carcinoma. Breast. 2008;17(4):341-6.

2. Marginean F, Rakha EA, Ho BC, Ellis IO, Lee AH. Histological features of medullary carcinoma and prognosis in triple-negative basal-like carcinomas of the breast. Mod Pathol. 2010;23(10):1357-63.

3. Kurozumi S, Matsumoto H, Kurosumi M, Inoue K, Fujii T, Horiguchi J, Shirabe K, Oyama T, Kuwano H. Prognostic significance of tumour-infiltrating lymphocytes for oestrogen receptor-negative breast cancer without lymph node metastasis. Oncol Lett. 2019;17(3):2647-56.

4. Ali HR, Provenzano E, Dawson SJ, Blows FM, Liu B, Shah M, Earl HM, Poole CJ, Hiller L, Dunn JA, et al. Association between CD8+ T-cell infiltration and breast cancer survival in 12,439 patients. Ann Oncol. 2014;25(8):1536-43.

5. Mahmoud SM, Paish EC, Powe DG, Macmillan RD, Grainge MJ, Lee AH, Ellis IO, Green AR. Tumor-infiltrating CD8+ lymphocytes predict clinical outcome in breast cancer. J Clin Oncol. 2011:29(15):1949-55.

6. Matkowski R, Gisterek I, Halon A, Lacko A, Szewczyk K, Staszek U, Pudelko M, Szynglarewicz B, Szelachowska J, Zolnierek A, Kornafel J. The prognostic role of tumor-infiltrating CD4 and CD8 T lymphocytes in breast cancer Anticancer Res. 2009:29(7):2445-51.

7. Macchetti AH, Marana HR, Silva JS, de Andrade JM, Ribeiro-Silva A, Bighetti S. Tumor-infiltrating CD4+ T lymphocytes in early breast cancer reflect lymph node involvement. Clinics (Sao Paulo). 2006;61(3):203-8.

8. Schmidt M, Weyer-Elberich V, Hengstler JG, Heimes AS, Almstedt K, GerholdAy A, Lebrecht A, Battista MJ, Hasenburg A, Sahin U, et al. Prognostic impact of CD4-positive T cell subsets in early breast cancer: a study based on the FinHer trial patient population. Breast Cancer Res. 2018:20(1):15-X.

9. Huang Y, Ma C, Zhang Q, Ye J, Wang F, Zhang Y, Hunborg P, Varvares MA, Hoft DF, Hsueh EC, Peng G. CD4+ and CD8+ T cells have opposing roles in breast cancer progression and outcome. Oncotarget. 2015;6(19):17462-78.

10. Janssen EM, Lemmens EE, Wolfe $T$, Christen U, von Herrath MG Schoenberger SP. CD4+ T cells are required for secondary expansion and memory in CD8+ T lymphocytes. Nature. 2003;421(6925):852-6.

11. Jamiyan T, Kuroda H, Yamaguchi R, Nakazato Y, Noda S, Onozaki M, Abe A, Hayashi M. Prognostic impact of a tumor-infiltrating lymphocyte subtype in triple negative cancer of the breast. Breast Cancer. 2020;27:880-92.

12. Mohammed ZM, Going JJ, Edwards J, Elsberger B, Doughty JC, MCMillan DC The relationship between components of tumour inflammatory cell infiltrate and clinicopathological factors and survival in patients with primary operable invasive ductal breast cancer. Br J Cancer. 2012;107(5): 864-73

13. Mahmoud SM, Lee AH, Paish EC, Macmillan RD, Ellis IO, Green AR. The prognostic significance of $B$ lymphocytes in invasive carcinoma of the breast. Breast Cancer Res Treat. 2012:132(2):545-53.

14. Song $H_{\text {, }}$ Heo SH, Bang WS, Park HS, Park IA, Kim YA, Park SY, Roh J, Gong G, Lee $\mathrm{HJ}$. Predictive value of tertiary lymphoid structures assessed by high endothelial Venule counts in the Neoadjuvant setting of triple-negative breast Cancer. Cancer Res Treat. 2017;49(2):399-407.

15. Schmidt M, Micke P, Gehrmann M, Hengstler JG. Immunoglobulin kappa chain as an immunologic biomarker of prognosis and chemotherapy response in solid tumors. Oncoimmunology. 2012;1(7):1156-8.

16. $X u Y$, Lan $S$, Zheng Q. Prognostic significance of infiltrating immune cell subtypes in invasive ductal carcinoma of the breast. Tumori. 2018;104(3): 196-201.

17. Mohammed ZM, Going JJ, Edwards J, MCMillan DC. The role of the tumour inflammatory cell infiltrate in predicting recurrence and survival in patients with primary operable breast cancer. Cancer Treat Rev. 2012:38(8):943-55.

18. Miligy IM, Toss MS, Khout $H$, Whisker L, Burrell HC, Ellis IO, Green AR, Macmillan D, Rakha EA. Surgical management of ductal carcinoma in situ of the breast: a large retrospective study from a single institution. Breast J. 2019;25(6):1143-53.

19. Mohammed ZM, Going JJ, Edwards J, Elsberger B, McMillan DC. The relationship between lymphocyte subsets and clinico-pathological determinants of survival in patients with primary operable invasive ductal breast cancer. Br J Cancer. 2013;109(6):1676-84.
20. Schmidt M, Bohm D, von Torne C, Steiner E, Puhl A, Pilch H, Lehr HA, Hengstler JG, Kolbl H, Gehrmann M. The humoral immune system has a key prognostic impact in node-negative breast cancer. Cancer Res. 2008;68(13): 5405-13.

21. Nielsen JS, Sahota RA, Milne K, Kost SE, Nesslinger NJ, Watson PH, Nelson $\mathrm{BH}$. CD20+ tumor-infiltrating lymphocytes have an atypical CD27- memory phenotype and together with CD8+ T cells promote favorable prognosis in ovarian cancer. Clin Cancer Res. 2012;18(12):3281-92

22. Garnelo M, Tan A, Her Z, Yeong J, Lim CJ, Chen J, Lim KH, Weber A, Chow $P$, Chung $A$, et al. Interaction between tumour-infiltrating B cells and T cells controls the progression of hepatocellular carcinoma. Gut. 2017;66(2):34251

23. Shi JY, Gao Q, Wang ZC, Zhou J, Wang XY, Min ZH, Shi YH, Shi GM, Ding ZB, Ke AW, et al. Margin-infiltrating CD20(+) B cells display an atypical memory phenotype and correlate with favorable prognosis in hepatocellular carcinoma. Clin Cancer Res. 2013;19(21):5994-6005.

24. Edin S, Kaprio T, Hagstrom J, Larsson P, Mustonen H, Bockelman C, Strigard K, Gunnarsson U, Haglund C, Palmqvist R. The prognostic importance of CD20(+) B lymphocytes in colorectal Cancer and the relation to other immune cell subsets. Sci Rep. 2019;9(1):19997-8.

25. Sautes-Fridman C, Lawand M, Giraldo NA, Kaplon H, Germain C, Fridman WH, Dieu-Nosjean MC. Tertiary lymphoid structures in cancers: prognostic value, regulation, and manipulation for therapeutic intervention. Front Immunol. 2016:7:407.

26. Hori S, Nomura T, Sakaguchi S. Control of regulatory T cell development by the transcription factor Foxp3. Science. 2003;299(5609):1057-61.

27. Ghiringhelli F, Larmonier N, Schmitt E, Parcellier A, Cathelin D, Garrido C, Chauffert B, Solary E, Bonnotte B, Martin F. CD4+CD25+ regulatory T cells suppress tumor immunity but are sensitive to cyclophosphamide which allows immunotherapy of established tumors to be curative. Eur J Immunol. 2004;34(2):336-44

28. Sakaguchi S, Miyara M, Costantino CM, Hafler DA. FOXP3+ regulatory T cells in the human immune system. Nat Rev Immunol. 2010;10(7):490-500.

29. Bohling SD, Allison $\mathrm{KH}$. Immunosuppressive regulatory $T$ cells are associated with aggressive breast cancer phenotypes: a potential therapeutic target. Mod Pathol. 2008;21(12):1527-32.

30. Liu F, Lang R, Zhao J, Zhang X, Pringle GA, Fan Y, Yin D, Gu F, Yao Z, Fu L. CD8(+) cytotoxic T cell and FOXP3(+) regulatory $T$ cell infiltration in relation to breast cancer survival and molecular subtypes. Breast Cancer Res Treat. 2011;130(2):645-55.

31. Bates GJ, Fox SB, Han C, Leek RD, Garcia JF, Harris AL, Banham AH. Quantification of regulatory T cells enables the identification of high-risk breast cancer patients and those at risk of late relapse. J Clin Oncol. 2006; 24(34):5373-80.

32. Allison $\mathrm{KH}$, Hammond MEH, Dowsett M, McKernin SE, Carey LA, Fitzgibbons PL, Hayes DF, Lakhani SR, Chavez-MacGregor M, Perlmutter J, et al. Estrogen and Progesterone Receptor Testing in Breast Cancer: ASCO/CAP Guideline Update. J Clin Oncol. 2020;38(12):1346-66.

33. Wolff AC, Hammond MEH, Allison KH, Harvey BE, Mangu PB, Bartlett JMS, Bilous M, Ellis IO, Fitzgibbons P, Hanna W, et al. Human epidermal growth factor receptor 2 testing in breast Cancer: American Society of Clinical Oncology/College of American Pathologists Clinical Practice Guideline Focused Update. Arch Pathol Lab Med. 2018;142(11):1364-82.

34. Tashima R, Nishimura R, Osako T, Nishiyama Y, Okumura Y, Nakano M, Fujisue M, Toyozumi Y, Arima N. Evaluation of an optimal cut-off point for the Ki-67 index as a prognostic factor in primary breast Cancer: a retrospective study. PLoS One. 2015;10(7):e0119565.

35. Hendry S, Salgado R, Gevaert T, Russell PA, John T, Thapa B, Christie M, van de Vijver K, Estrada MV, Gonzalez-Ericsson PI, et al. Assessing Tumorinfiltrating Lymphocytes in Solid Tumors: A Practical Review for Pathologists and Proposal for a Standardized Method From the International Immunooncology Biomarkers Working Group: Part 1: Assessing the Host Immune Response, TILs in Invasive Breast Carcinoma and Ductal Carcinoma In Situ, Metastatic Tumor Deposits and Areas for Further Research. Adv Anat Pathol. 2017;24(5):235-51.

36. Loi S, Drubay D, Adams S, Pruneri G, Francis PA, Lacroix-Triki M, Joensuu $H_{\text {, }}$ Dieci MV, Badve S, Demaria S, et al. Tumor-infiltrating lymphocytes and prognosis: a pooled individual patient analysis of early-stage triple-negative breast cancers. J Clin Oncol. 2019;37(7):559-69.

37. Bruno TC, Ebner PJ, Moore BL, Squalls OG, Waugh KA, Eruslanov EB, Singhal S, Mitchell JD, Franklin WA, Merrick DT, et al. Antigen-presenting 
Intratumoral B cells affect CD4(+) TIL phenotypes in non-small cell lung Cancer patients. Cancer Immunol Res. 2017;5(10):898-907.

38. Rivera A, Chen CC, Ron N, Dougherty JP, Ron Y. Role of B cells as antigenpresenting cells in vivo revisited: antigen-specific $B$ cells are essential for $T$ cell expansion in lymph nodes and for systemic $T$ cell responses to low antigen concentrations. Int Immunol. 2001;13(12):1583-93.

39. Rossetti RAM, Lorenzi NPC, Yokochi K, Rosa MBSF, Benevides L, Margarido PFR, Baracat EC, Carvalho JP, Villa LL, Lepique AP. B lymphocytes can be activated to act as antigen presenting cells to promote anti-tumor responses. PLoS One. 2018;13(7):e0199034.

40. Rubtsov AV, Rubtsova K, Kappler JW, Jacobelli J, Friedman RS, Marrack P. CD11c-expressing B cells are located at the T cell/B cell border in spleen and are potent APCs. J Immunol. 2015;195(1):71-9.

41. Yanaba K, Bouaziz JD, Matsushita T, Magro CM, St Clair EW, Tedder TF. Blymphocyte contributions to human autoimmune disease. Immunol Rev. 2008;223:284-99

42. Zarkhin V, Chalasani G, Sarwal MM. The yin and yang of B cells in graft rejection and tolerance. Transplant Rev (Orlando). 2010;24(2):67-78.

43. Takemura S, Braun A, Crowson C, Kurtin PJ, Cofield RH, O'Fallon WM Goronzy JJ, Weyand CM. Lymphoid neogenesis in rheumatoid synovitis. J Immunol. 2001;167(2):1072-80.

44. Kang YM, Zhang X, Wagner UG, Yang H, Beckenbaugh RD, Kurtin PJ, Goronzy JJ, Weyand CM. CD8 T cells are required for the formation of ectopic germinal centers in rheumatoid synovitis. J Exp Med. 2002;195(10): 1325-36.

45. Gheybi MK, Farrokhi S, Ravanbod MR, Ostovar A, Mehrzad V, Nematollahi P. The correlation of CD19+CD24 + CD38 + B cells and other clinicopathological variables with the proportion of circulating Tregs in breast cancer patients. Breast Cancer. 2017;24(6):756-64.

46. Chen LC, Delgado JC, Jensen PE, Chen X. Direct expansion of human allospecific FoxP3+CD4+ regulatory T cells with allogeneic B cells for therapeutic application. J Immunol. 2009;183(6):4094-102.

47. Tu W, Lau YL, Zheng J, Liu Y, Chan PL, Mao H, Dionis K, Schneider P, Lewis DB. Efficient generation of human alloantigen-specific $C D 4+$ regulatory $T$ cells from naive precursors by CD40-activated B cells. Blood. 2008;112(6): 2554-62.

48. Ahmadzadeh M, Felipe-Silva A, Heemskerk B, Powell DJ, Wunderlich JR, Merino MJ, Rosenberg SA. FOXP3 expression accurately defines the population of intratumoral regulatory $T$ cells that selectively accumulate in metastatic melanoma lesions. Blood. 2008;1 12(13):4953-60.

49. Kryczek I, Liu R, Wang G, Wu K, Shu X, Szeliga W, Vatan L, Finlayson E, Huang E, Simeone D, et al. FOXP3 defines regulatory T cells in human tumor and autoimmune disease. Cancer Res. 2009;69(9):3995-4000.

50. Amgad M, Stovgaard ES, Balslev E, Thagaard J, Chen W, Dudgeon S, Sharma A, Kerner JK, Denkert C, Yuan Y, et al. International Immuno-oncology biomarker working group: report on computational assessment of tumor infiltrating lymphocytes from the international Immuno-oncology biomarker working group. NPJ Breast Cancer. 2020;6:16-2.

51. Wouters MCA, Nelson BH. Prognostic significance of tumor-infiltrating $B$ cells and plasma cells in human Cancer. Clin Cancer Res. 2018;24(24):612535.

\section{Publisher's Note}

Springer Nature remains neutral with regard to jurisdictional claims in published maps and institutional affiliations.

Ready to submit your research? Choose BMC and benefit from:

- fast, convenient online submission

- thorough peer review by experienced researchers in your field

- rapid publication on acceptance

- support for research data, including large and complex data types

- gold Open Access which fosters wider collaboration and increased citations

- maximum visibility for your research: over $100 \mathrm{M}$ website views per year

At BMC, research is always in progress.

Learn more biomedcentral.com/submissions 